\title{
Elementos importantes de la cadena de abastecimiento del sector automotriz en Colombia
}

\section{Important elements of the automotive supply chain in Colombia}

\author{
DOI: http://dx.doi.org/10.17981/ingecuc.15.1.2019.15
}

Artículo de Investigación Científica. Fecha de Recepción: 29/10/2018. Fecha de Aceptación: 05/04/2019

\author{
Gilma Rocío Peña-Meneses \\ Corporación Minuto de Dios. Bogotá (Colombia) \\ rpena@uniminuto.edu.co
}

\author{
Rafael Guillermo García-Cáceres \\ Universidad Pedagógica y Tecnológica de Colombia. Sogamoso (Colombia) \\ rafael.garcia01@uptc.edu.co
}

Para citar este artículo:

Gilma Peña-Meneses; Rafael García-Caceres "Elementos importantes de la cadena de abastecimiento del sector automotriz en Colombia", INGE CUC, vol. 15, no. 1, pp. 168-183, 2019. DOI: http://doi.org/10.17981/ingecuc.15.1.2019.15

\section{Resumen}

Introducción- La globalización ha sido un aspecto principal para el desarrollo del paradigma de la Cadena de Abastecimiento (CA), la cual busca minimizar los costos de producción, y maximizar la oferta de bienes con ventajas competitivas, en términos de economías de escala y mano de obra. La industria automotriz es objeto de estudio permanente, debido al contexto competitivo de la industria en crecimiento en el tiempo, por diversos factores incluyendo: el impacto ambiental, la movilidad, la personalización, el incremento de competencia directa e indirecta, los cambios sociales y el manejo del riesgo.

Objetivo- El presente artículo busca destacar la importancia y caracterizar la CA del sector automotriz de un país en desarrollo como es el caso de Colombia.

Metodología- El estudio se realizó a través de una metodología compuesta de los siguientes pasos: Paso 0. Proporcionar el contexto económico y social a nivel global y doméstico de la CA; Paso 1. Identificar las etapas, escalones y enlaces de la CA; Paso 2. Describir el valor agregado de los agentes y componentes de la CA; Paso 3. Describir las especificidades del desempeño de la CA; y Paso 4. Diagnóstico y conclusiones sobre la condición de la CA.

Resultados- El sector automotriz y de motos constituyen el $4 \%$ de la producción bruta industrial colombiana y la ciudad capital es el principal contribuyente a la generación de empleo a nivel nacional, además de concentrarse en tres grandes ejes: ensamble de vehículos, producción de autopartes y ensamble de motocicletas.

Conclusiones-Se evidencian dos cadenas importantes para el sector automoriz; la importación de vehículos terminados (ensamble fuera de Colombia) y automotores ensamblados dentro del país. Y para Colombia el principal reto de la distribución de las marcas de importados es el soporte de producto, servicio postventa y repuestos en toda la geografía nacional.

Palabras clave- Caracterización; cadena de abastecimiento; sector automotriz.

\begin{abstract}
Introduction- Globalization has been a major aspect for the development of the Supply Chain (SC) paradigm, which seeks to minimize production costs and maximize the supply of goods with competitive advantages, in terms of economies of scale and labor. The automotive industry is the object of permanent study, due to the competitive context of the industry growth over time, by various factors including: environmental impact, mobility, customization, increased direct and indirect competition, social changes and risk management.
\end{abstract}

Objective- This project seeks to highlight the importance and characterize the CA of the automotive sector in a developing country such as Colombia.

Method-The study was carried out using a methodology composed of the following steps: Step 0. Provide the global and domestic economic and social context of the CA; Step 1. Identify the stages, steps and linkages of the CA; Step 2. Describe the added value of CA agents and components; Step 3. Describe the specificities of CA performance; and Step 4. Diagnosis and conclusions about the condition of the CA.

Results-The automotive and motorcycle sectors account for $4 \%$ of Colombia's gross industrial production and the capital city is the main contributor to the generation of employment at the national level, in addition to concentrating on three major axes: vehicle assembly, production of auto parts and assembly of motorcycles.

Conclusions- There are two important chains for the automotive sector; the import of finished vehicles (assembly outside Colombia) and automobiles assembled within the country. And for Colombia the main challenge of the distribution of imported brands is the product support, after-sales service and spare parts throughout the national geography.

Keywords- Characterization; supply chain; automotive sector. 


\section{INTRODUCCIÓN}

Según Chopra [2], la CA está conformada por todos aquellos agentes, desde el primer proveedor, involucrados de manera directa o indirecta en la satisfacción de los requerimientos del cliente final. La CA incluye no solamente al fabricante y al proveedor, sino también a los transportadores, almacenistas, vendedores al detalle (o menudeo), organizaciones gubernamentales y no gubernamentales, con y sin ánimo de lucro, e incluso a los mismos clientes. El modelo macro de la $\mathrm{CA}$ es replicado por cada uno de los agentes, con las mismas etapas (aprovisionamiento-producción-distribución) de la CA, y en conjunto permiten satisfacer los requerimientos del cliente. Estas funciones incluyen, pero no están limitadas al desarrollo de nuevos productos; la mercadotecnia, las operaciones, la distribución, las finanzas y el servicio al cliente. El mayor logro de la CA es conseguir a cada eslabón obtener un alto grado de integración, coordinación y articulación; esto sucede cuando las especificaciones del producto son uniformes y el aprovisionamiento de los insumos es global [3].

La globalización ha sido un aspecto principal para el desarrollo del paradigma de la CA. La globalización busca minimizar los costos de producción y maximizar la oferta de bienes, en otras palabras, busca que cada uno de los suministros sea adquirido en lugares con réditos de existencias y con ventajas competitivas en términos de economías de escala y mano de obra; lo cual trae como resultado que las ventajas competitivas de tiempo y lugar cobren cada vez mayor relevancia, y en consecuencia, las compañías se apoyen en la logística como función generadora de valor empresarial. Por esto, la gestión de la CA se enfoca en la búsqueda de estrategias que permitan la competitividad y sostenibilidad empresarial a través de mejores costos y niveles de servicio.

La industria automotriz es objeto de estudio permanente, debido a su importancia como generador de empleo y su peso como dinamizador de la economía, y se considera como uno de los más importantes sectores industriales. Como indican algunos autores [3], la industria se caracteriza por el alto nivel de complejidad, tanto en producción como en logística; una compañía diseña y produce alrededor de 15.000 partes que componen un vehículo, otras piezas son suministradas por proveedores directos, aproximadamente 600 en todo el mundo, los cuales también pueden tener otros sub-proveedores. La red logística y de producción involucrada por lo tanto requiere de una gestión altamente controlada y coordinada, soportada por recursos de múltiple naturaleza, tecnología y personal capacitado.

Así, el contexto competitivo de la industria se ha incrementado en el tiempo debido a diversos factores como son: impacto ambiental, movilidad, personalización, incremento de competencia directa e indirecta y cambios sociales, entre otros [3], así mismo, el manejo de los riesgos es una variable que afecta los temas de producción en la cadena de abastecimiento [4], un $37 \%$ de las empresas automotrices no gestionan prácticas para minimizar el riesgo, marcandolo como un desafío importante en la predicción de puntos de falla y en el impacto generado por éste para la industria.

En lo que atañe a Colombia [5], el país tiene un escenario "ideal" para generar una plataforma de fabricación y ensamble de vehículos, camiones, buses y autopartes, a causa de su estratégica localización. Pese a ello, su competitividad es baja debido al tamaño del mercado, presionando la necesidad de desarrollar una mayor influencia comercial que pueda generar la demanda regional suficiente para desarrollar las economías de escala necesarias y competir a nivel global. El sector de autopartes y ensamble de vehículos es uno de los 16 sectores que hacen parte del Programa de Transformación Productiva del país, para conseguir el reconocimiento de Colombia en el 2032 como un país líder exportador en el mercado de autopartes por generar ingresos anuales del orden de los USD 10 mil millones.

Actualmente, los incentivos o programas que existen en la industria para motivar y promover el sector son: Programa de fomento para la industria Automotor (PROFIA), Régimen de ensamble para la industria automotor en el marco de la CAN, Depósitos habilitados para la transformación (DHTE), Uso de zonas francas para ensamble, Ensamble de vehículos amigables con el ambiente y por último, el convenio automotor Colombia-Brasil. [5].

El presente trabajo presenta la caracterización de la CA del sector automotriz en Colombia, y para lo cual hace uso de la metodología planteada [1], al identificar y describir el valor de los agentes y eslabones involucrados de la CA.

\section{Estado DEL Arte}

La literatura sobre CA muestra una serie de trabajos que incluyen al sector de medicamentos en Bogotá y varias CAs agroindustriales: café [6], cacao [1] y palma [7]. La metodología usada en dichos trabajos fue inicialmente inspirada en trabajos como [8] y [9]. La más reciente versión de caracterización de CA presentada en un trabajo [10] muestra una metodología con unas pautas seguidas en este trabajo. Y en lo que atañe a estudios de caso a nivel país se encontró con la siguiente literatura:

En el año 2012 Survey ISO mostró el resultado de un estudio sobre el número de empresas certificadas con el estándar internacional, en el cual Colombia ocupo el cuarto puesto en Centro y Sur América con 74 certificaciones después de Argentina, Brasil y Chile [11]. 
La gestión de los proveedores de primer nivel, encargados de abastecer una parte o alguno de los sistemas principales del automóvil, suministran directamente sus productos a los ensambladores de automóviles; esto ha sido más estudiado que los de segundo nivel, quienes suministran sus productos directamente a los proveedores de primer nivel. Con relación a este último caso se encuentran trabajos [12] que identifican las relaciones cliente-proveedor, para el caso mexicano las caracteriza de dos maneras: una de simple intercambio comercial, en donde se busca mantener una buena relación comercial con relaciones a corto plazo y no se ve como una ventaja competitiva, y otra como socios estratégicos en donde la finalidad es convertir a los proveedores en socios que sirvan como una ventaja competitiva en el mercado. El estudio concluye con dos consideraciones importantes para las empresas ensambladoras a la hora de escoger sus proveedores; por un lado el precio y por el otro la calidad, es decir, siguen imperando los aspectos productivos para ciertas decisiones. Se logra concluir que la cooperación y la confianza entre los proveedores de nivel 1 y las ensambladoras es la tendencia general en la industria automotriz en México y la comunicación en los dos sentidos ha mejorado las relaciones contractuales entre los actores de la CA.

En otros casos [13], las ensambladoras japonesas han aumentado, no obstante, sus proveedores han decrecido moderadamente; tal vez por los estándares de calidad exigidos. El rápido crecimiento de la industria China hace parecer que la industria de automóviles en Japón, América y Europa no crece. Por su parte, el auge de los automóviles híbridos y eléctricos probablemente cambien la estructura de la industria y su CA, esto se evidencia en las innovaciones que vienen realizando los proveedores de llantas, baterías y carrocerías, con los cambios tecnológicos más profundos de la industria, de hecho, algunos estudios ya inician investigación sobre la adopción de prácticas de gestión sostenible en la cadena de suministro automotriz, las cuales aseguran [14] son más difíciles de implementar en las económicas emergentes.

En un intento por disminuir costos y defender el mercado, debido a la arremetida de las empresas japonesas, los fabricantes de Estados Unidos decidieron llevar su producción a países vecinos como Canadá y México. Al respecto, [15] decidieron investigar sobre innovación en el sector automotriz con entornos económicos poco favorables, para ello plantearon el trabajo en varias regiones de México, encontrando como la mayoría de las empresas proveedoras de autopartes son subsidiadas por empresas extranjeras; los proveedores de autopartes se limitan a seguir los requerimientos de las plantas de ensamblaje, provocando una gran inversión en contratación de personal experto y adquisición de tecnología de punta, ocasionando que pocas empresas se mantengan con capital $100 \%$ mexicano. Las empresas extranjeras no hacen estudios de innovación y desarrollo en México, solo ven lo atractivo de la mano de obra económica y la conveniente situación geográfica de estas empresas. En los últimos 5 años, los únicos procesos de innovación realizados se relacionaron con procesos de producción y diseño de productos [15].

También [16] se analiza la relación entre la dinámica de crecimiento de la producción de la manufactura y el sector automotriz en las entidades federativas de la frontera norte de México (Baja California, Sonora, Chihuahua, Coahuila, Nuevo León y Tamaulipas). Los resultados conseguidos sugieren que el sector automotriz, sin ser el único factor, está empujando el crecimiento manufacturero en la zona norte del país, generando importantes beneficios, en variables como empleo, producción y calificación de mano de obra, entre otros.

Por otro lado [17], estudios de la resiliencia en la CA del sector automotriz en Brasil, han probado como en los últimos años el crecimiento de la demanda de vehículos en Brasil ha seguido un ritmo marcado, por la capacidad de oferta adquirida de los proveedores, razón por la cual el país posee un alto riesgo de interrupciones en la CA. Cuando un eslabón de la CA, en este caso un proveedor, tiene problemas de capacidad para cumplir con la demanda, afecta a los otros eslabones de la CA, es allí donde el proveedor debe tener la capacidad para responder a estos imprevistos y dar soluciones para no afectar la CA del fabricante.

En otros casos [18], desarrollan un modelo de dinámica de sistemas para la evaluación del desempeño de las CAs de suministro operando en mercados emergentes. El modelo considera las características de las operaciones automotrices en países con rápido crecimiento y la importancia de estos territorios en el desarrollo futuro de la industria automotriz. Dentro de las conclusiones se propone la necesidad de tener procesos logísticos mejor organizados y la necesidad de integrar dinámicamente las ventajas diferenciales entre cada uno de los actores (proveedores, fabricantes, etc.).

Los proveedores de segundo nivel también son estudiados [19], como el análisis del caso de Brasil, donde se centran en las relaciones y la integración de procesos entre cuatro proveedores de primer nivel y sus principales proveedores de segundo nivel. $\mathrm{Al}$ respecto, a principios de los años 90 el foco de la inversión en Brasil se dio en el sector automotriz, en la modernización de tecnológica y la construcción de nuevas plantas, dando como resultado que los principales fabricantes de automóviles en el mundo tengan planta de fabricación en ese país, situandolo como uno de los mayores países ensambladores de autos en Latinoamérica. Además, los proveedores de segundo nivel son auditados periódicamente por los proveedores de primer nivel 
para evitar inconformidades, y de esta manera se escogen aquellos proveedores con certificados de sistemas de gestión de calidad ISO 9001.

\section{III.MetodoloGía}

A través de una investigación documental, en donde se utilizan fuentes primarias de bases de datos científicas y páginas gubernamentales, este trabajo emplea una metodología formalizada [1], Los pasos de la metodología son:

Paso 0. Proporcionar el contexto económico y social a nivel global y doméstico de la CA.

a. Contexto Mundial.

b. Contexto Nacional.

Paso 1. Identificar las etapas, escalones y enlaces de la CA.

a. Eslabón de Aprovisionamiento.

b. Marcas (ensambladoras).

c. Eslabón de Producción

d. Eslabón de Distribución

e. Operadores logísticos

Paso 2. Describir el valor agregado de los agentes y componentes de la CA.

a. Eslabón de Aprovisionamiento

b. Eslabón de Producción

c. Eslabón de Distribución

d. Cadena de abastecimiento de vehículos en Colombia

Paso 3. Describir las especificidades del desempeño de la CA.

Paso 4. Diagnóstico y conclusiones sobre la condición de la CA.

\section{Desarrollo}

Paso 0. Proporcionar el contexto económico y social a nivel global y doméstico de la CA.

\section{A. Contexto Mundial}

Pese a que el inicio del automóvil se origina en Europa, es en Estados Unidos donde la industria automotriz se consolida debido al tamaño del mercado y a la reducción de los costos de producción con la introducción de la producción en serie. El surgimiento de fabricantes, conocidos, como los "tres grandes" (Ford, General Motors y Chrysler) aceleró el proceso de fabricación y exportación de automóviles a diferentes partes del mundo, a lo cual siguieron Japón y algunos países europeos, como Alemania y Francia, y más recientemente otros países como Corea del Sur.

Desde el punto de vista global el sector industrial en general y el automotriz en particular ha mostrado un crecimiento significativo y una evolución acelerada que ha impactado de manera importante la economía mundial desde los años 70 , con algunos altibajos a través de los años [21]. La contribución global de la industria automotriz a la producción es de aproximadamente 2.6 trillones de dólares [22] y para el año 2012 la venta global de vehículos supero los 84 millones, en este mismo año, 9 millones de trabajadores fueron contratados de forma directa y 50 millones de puestos de trabajo en forma indirecta. El valor de la producción de esta industria equivale a una sexta parte de la economía mundial [23].

La crisis financiera [19] que enfrentó Estados unidos, enmarcada por la recesión económica a mediados del 2008, afectaron el sector automotor mundial [24]. Variables como el aumento del precio del petróleo desde el año 2004 ocasionaron una baja en la adquisición de vehículos deportivos y pickups, al igual, la decisión de las gerencias de empresas de los fabricantes americanos al otorgar a sus sindicatos sueldos mayores, crearon desigualdad respecto a las partes no sindicalizadas y una estructura de costos no competitiva [25].

Estados Unidos, Japón, China e India han intentado mantener sus posiciones y ampliar sus ventas, realizando avances tecnológicos que lleven a minimizar costos y producir a menor precio. El gobierno de Estados Unidos ha hecho esfuerzos por fortalecer con incentivos económicos; como medida para reactivar la economía y el empleo tras la crisis financiera global, desencadenada desde este país por la caída financiera en el sector inmobiliario. La crisis que afectó a casi todos los sectores de la economía americana tuvo un significativo impacto mundial, en donde el sector automotor se vio fuertemente afectado. El alto costo de las materias primas y la falta de corrección en el consumo de combustible de los automóviles, crearon en el consumidor la renuencia de adquirir vehículos fabricados en Estados Unidos, y a cambio favorecieron la adquisición de autos japoneses, coreanos e incluso europeos, ganando segmentos importantes del mercado [24]

En respuesta, las empresas estadounidenses establecieron nuevas líneas de producción en otros países con menores costos de mano de obra, como México, para disminuir costos; aunque en el corto plazo estimuló la producción de vehículos, al mediano plazo no ha logrado establecer un estándar de competitividad más consistente respecto a las compañías de automóviles asiáticas y europeas [26].

A continuación, la tabla 1 muestra la dinámica de unidades producidas desde el año 2012, por los 10 principales países productores en el mundo, estas cifras incluyen vehículos de pasajeros, microbuses, camiones y autobuses, aunque por muchas décadas Estados unidos ha liderado la producción de automóviles, vemos como China ha liderado la producción en los últimos años y sigue en crecimiento como se evidencia en el año 2017. 
TABla 1. Los 10 PaÍses MaYores PRODUCTORES DE AUTOMÓVILES EN EL MUNDO (CIFRAS EN MILLONES).

\begin{tabular}{|c|c|c|c|}
\hline País & $\begin{array}{c}\text { Unidades } \\
\text { producidas } \\
2012\end{array}$ & $\begin{array}{c}\text { Unidades } \\
\text { producidas } \\
2013\end{array}$ & $\begin{array}{c}\text { Unidades } \\
\text { producidas } \\
2014\end{array}$ \\
\hline China & 19.27 & 22.11 & 23.72 \\
\hline EUA & 10.33 & 11.06 & 11.66 \\
\hline Japón & 10.32 & 9.63 & 9.77 \\
\hline Alemania & 5.9 & 5.71 & 5.9 \\
\hline $\begin{array}{c}\text { Corea del } \\
\text { Sur }\end{array}$ & 4.55 & 4.52 & 4.52 \\
\hline Brasil & 3.40 & 3.71 & 3.14 \\
\hline India & 4.17 & 3.89 & 3.84 \\
\hline México & 3.001 & 3.05 & 3.36 \\
\hline Canadá & 2.46 & 2.37 & 2.39 \\
\hline Francia & 1.96 & 1.74 & 1.82 \\
\hline País & Unidades & $\begin{array}{c}\text { Unidades } \\
\text { producidas }\end{array}$ & $\begin{array}{c}\text { Unidades } \\
\text { producidas } \\
2017\end{array}$ \\
\hline China & 24.50 & 28.11 & 29.01 \\
\hline EUA & 12.10 & 12.19 & 11.18 \\
\hline Japón & 9.27 & 9.20 & 9.69 \\
\hline Alemania & 6.03 & 6.06 & 5.64 \\
\hline $\begin{array}{c}\text { Corea del } \\
\text { Sur }\end{array}$ & 4.55 & 4.22 & 4.11 \\
\hline Brasil & 2.42 & 2.15 & 2.69 \\
\hline India & 4.12 & 4.48 & 4.78 \\
\hline México & 3.56 & 3.59 & 4.06 \\
\hline Canadá & 2.28 & 2.37 & 2.19 \\
\hline Francia & 1.97 & 2.08 & 2.22 \\
\hline
\end{tabular}

Fuente: Elaboración propia con datos [20].

Como se nota, la producción está marcada por las economías de escala y está centrada a países que cuentan con altas demandas, propias o regionales, o que tienen una marcada política exportadora, como Japón o Alemania donde la denominación "hecho en ...., es reconocida como sello de calidad para apalancar la reputación y el atractivo de la demanda. Con relación a la venta de autos, la situación es similar, China continua con el primer puesto seguido de Estados Unidos, Japón y Alemania; aunque India supera las ventas de Alemania en los últimos dos años. La tabla 2 muestra el historial de ventas desde 2012 hasta 2017.

La crisis energética propicio que en la década de los años 90 aparecieran los automóviles con moto-
TABla 2. Los 10 Países MaYoRes

COMERCIALIZADORES DE AUTOMÓVILES EN EL MUNDO.

\begin{tabular}{|c|c|c|c|}
\hline País & $\begin{array}{l}\text { Unidades } \\
\text { vendidas } \\
2012\end{array}$ & $\begin{array}{c}\text { Unidades } \\
\text { vendidas } \\
2013\end{array}$ & $\begin{array}{l}\text { Unidades } \\
\text { vendidas } \\
2014\end{array}$ \\
\hline China & 19.306 .435 & 21.984 .079 & 23.499 .001 \\
\hline $\begin{array}{l}\text { Estados } \\
\text { Unidos }\end{array}$ & 14.785 .936 & 15.883 .443 & 16.522 .663 \\
\hline Japón & 5.369 .721 & 5.375 .513 & 5.562 .888 \\
\hline Alemania & 3.394 .002 & 3.257 .718 & 3.356 .718 \\
\hline Brasil & 3.802 .071 & 3.767 .370 & 3.498 .012 \\
\hline India & 3.595 .508 & 3.241 .302 & 3.177 .005 \\
\hline Francia & 2.331 .731 & 2.207 .373 & 2.210 .927 \\
\hline Canadá & 1.716 .178 & 1.780 .523 & 1.890 .387 \\
\hline $\begin{array}{l}\text { Corea del } \\
\text { Sur }\end{array}$ & 1.532 .087 & 1.543 .564 & 1.661 .868 \\
\hline México & 1.024 .574 & 1.100 .542 & 1.135 .409 \\
\hline País & $\begin{array}{c}\text { Unidades } \\
\text { vendidas } \\
2015\end{array}$ & $\begin{array}{c}\text { Unidades } \\
\text { vendidas } \\
2016\end{array}$ & $\begin{array}{c}\text { Unidades } \\
\text { vendidas } \\
2017\end{array}$ \\
\hline China & 24.597 .583 & 28.028 .175 & 29.122 .530 \\
\hline $\begin{array}{l}\text { Estados } \\
\text { Unidos }\end{array}$ & 17.470 .659 & 17.865 .773 & 17.583 .841 \\
\hline Japón & 5.046 .511 & 4.970 .260 & 5.238 .888 \\
\hline Alemania & 3.539 .825 & 3.708 .867 & 3.811 .246 \\
\hline Brasil & 2.568 .976 & 2.050 .321 & 2.238 .914 \\
\hline India & 3.425 .336 & 3.669 .277 & 4.017 .539 \\
\hline Francia & 2.345 .092 & 2.478 .472 & 2.604 .942 \\
\hline Canadá & 1.939 .949 & 1.983 .745 & 2.077 .000 \\
\hline $\begin{array}{l}\text { Corea del } \\
\text { Sur }\end{array}$ & 1.833 .786 & 1.823 .041 & 1.798 .795 \\
\hline México & 1.351 .648 & 1.647 .723 & 1.570 .764 \\
\hline
\end{tabular}

Fuente: Elaboración propia con datos [20].

res híbridos, debido posiblemente a la presión ambientalista (la mayoría de los fabricantes ya han iniciado la fabricación de estos modelos). Para señalar algunos casos, Toyota presentó su Prius en el mercado japonés en 1997, y Honda siguió con el lanzamiento de su primer vehículo hibrido "el Insight" en California en 1998 y luego en todo el mundo a un precio capaz de competir con los autos convencionales. Para el año 2005 las exportaciones de vehículos híbridos de Toyota, principalmente a los Estados Unidos, habían superado las ventas del mercado interno, al igual que Honda. Tesla motors 
en este rango de mercado se ha convertido en un representante muy innovador en la fabricación de automoviles eléctricos. La compañia fundada en 2003, con sede en California, se dio a conocer por comercializar el primer auto deportivo completamente eléctrico [27]. El éxito de la compañía radica en su enfoque innovador enfocado diseñar autos eléctricos de lujo, no obstante, actualmente empresas como BMW, Ferrari, Porshe y Audi están comenzando a presentar vehiculos con características similares a precios competitivos [28].

Respecto a las marcas que más se comercializan en el mundo, Toyota, seguido de la marca Volkswagen y muy de cerca Ford. La tabla 3 muestra el total de producción de las 10 marcas más vendidas a nivel mundial según datos 2017 . Y en la tabla 4 se presentan los países con mayor adquisición de automóviles por cada 1000 habitantes, evidenciando que ninguno de estos países es productor.

Tabla 3. Las 10 Marcas más VEndidas A NIVEL MUNDIAL EN EL 2017.

\begin{tabular}{|c|c|c|c|}
\hline Grupo & $\begin{array}{c}\text { Total de } \\
\text { producción }\end{array}$ & Grupo & $\begin{array}{c}\text { Total de } \\
\text { producción }\end{array}$ \\
\hline Toyota & 7.843 .423 & Chevrolet & 3.857 .388 \\
\hline Volkswagen & $6, .639 .250$ & Suzuki & 2.891 .415 \\
\hline Ford & 5.953 .122 & Mercedes & 2.511 .293 \\
\hline Nissan & 4.834 .694 & Kia & 2.511 .293 \\
\hline Hyundai & 3.951 .176 & Renault & 2.275 .227 \\
\hline
\end{tabular}

Fuente: Elaboración propia con datos [30].

Tabla 4. Principales Países CONSUmidores de aUtomóviles POR CADA 1000 HABITANTES EN EL MUNDO.

\begin{tabular}{|c|c|c|c|}
\hline País & $\begin{array}{c}\text { Automóviles } \\
\text { por cada } \\
\mathbf{1 0 0 0} \\
\text { habitantes }\end{array}$ & País & $\begin{array}{c}\text { Automóviles } \\
\text { por cada } \\
\mathbf{1 0 0 0} \\
\text { habitantes }\end{array}$ \\
\hline Australia & 556 autos & Islandia & 644 autos \\
\hline Malta & 579 autos & Luxemburgo & 665 autos \\
\hline $\begin{array}{c}\text { Nueva } \\
\text { Zelanda }\end{array}$ & 599 autos & Mónaco & 732 autos \\
\hline Italia & 602 autos & Liechtenstein & 750 autos \\
\hline $\begin{array}{c}\text { Puerto } \\
\text { Rico }\end{array}$ & 621 autos & San Marino & 1139 autos \\
\hline
\end{tabular}

Fuente: Elaboración propia con datos [31].

El mercado de los automóviles puede caracterizarse por los tipo de motores en tres grupos: el motor más económico con relativamente mayor consumo de combustible asociado al $30 \%$ a $35 \%$ de compradores, el segundo grupo dispuesto a pagar más dinero por un motor más potente y con menor consumo de combustible $60 \%$ a $65 \%$, y un tercer grupo en donde se encuentran pocos clientes, cerca de un $5 \%$, que buscan pagar un poco más por motores limpios y amigables con el planeta [29].

En Latinoamérica, el sector automotriz es clave para la economía de la región, por esta razón, algunos de sus países se consideran insertados en el movimiento global de producción de vehículos, dentro del grupo de países emergentes. Los países emergentes pasaron a ser el principal productor de vehículos en 2004, y a 2009 ya producían el doble de vehículos que los países tradicionales. En lo que atañe a la demanda, en América Latina la mayoría de países han visto incrementar su parque automotor en una proporción mayor a la del aumento de su población, se destacan los casos de Colombia y Perú con un crecimiento del parque automotor del $300 \%$ en el periodo 2000 - 2009.

Los dos grandes productores de autos en Latinoamérica son Brasil y México, potencias tanto en producción como en ventas, actualmente concentran el $90 \%$ de la producción de la región y están entre los 10 países que más producen automóviles en el mundo [32]. En la tabla 5 se muestra el comportamiento de la venta de vehículos nuevos por países en Latinoamérica en los últimos años 2015 y 2017, encontrando como México supera en producción a Brasil, sin embargo, Brasil está por encima de México en las ventas de automotores anuales.

Tabla 5. Comportamiento de PRoducción y Venta de vehículos POR PAÍSES EN LATINOAMÉRICA (UNIDADES).

\begin{tabular}{|c|c|c|c|}
\hline País & Ventas 2015 & $\begin{array}{c}\text { Producción } \\
2015\end{array}$ & $\begin{array}{c}\text { Ventas } \\
2016\end{array}$ \\
\hline Brasil & 2.568 .976 & 2.429 .463 & 2.050 .321 \\
\hline México & 1.351 .648 & 3.565 .469 & 1.647 .723 \\
\hline Argentina & 605.933 & 533.683 & 709.482 \\
\hline Colombia & 272.400 & 78.070 & 246.500 \\
\hline Ecuador & 82.600 & 4.800 & 63.555 \\
\hline Venezuela & 14.700 & 18.300 & 5.200 \\
\hline País & $\begin{array}{c}\text { Producción } \\
2016\end{array}$ & $\begin{array}{c}\text { Ventas } \\
2017\end{array}$ & $\begin{array}{c}\text { Producción } \\
2017\end{array}$ \\
\hline Brasil & 2.156 .356 & 2.238 .915 & 2.699 .672 \\
\hline México & 3.597 .462 & 1.570 .764 & 4.068 .415 \\
\hline Argentina & 472.776 & 900.403 & 472.158 \\
\hline Colombia & 79.036 & 233.960 & 74.994 \\
\hline Ecuador & 2.700 & 118.281 & 2700 \\
\hline Venezuela & 2.850 & 14.084 & 1.774 \\
\hline
\end{tabular}

Fuente: Elaboración propia con datos [20]. 
En la CA del sector automotriz se identifican las empresas por la función y aporte en la integración del automóvil. Estas se organizan por la forma como interactúan con los ensambladores de automóviles, ya sea directa o indirectamente, en un contexto de CA global se identifican los grandes fabricantes de automóviles [33]. Por su parte las ensambladoras y los distribuidores suelen representar un mercado nacional y regional y son regulados por la normatividad de cada país.

Los fabricantes de automóviles suelen hacer las adquisiciones de insumos a través de proveedores localizados en lugares cercanos con el fin de reducir los costos de transporte, sin embargo, cuando se trata de insumos claves la calidad se convierte en el criterio más importante de decisión. Al respecto, fabricantes como Toyota tiene como estrategia corporativa la creación de alianzas estratégicas con proveedores claves, como Johnson Controls o Aisin Seiki [34], empresas donde la cultura organizacional y el componente de innovación son compatibles con sus valores institucionales.

\section{B. Contexto Nacional}

El sector automotriz y de motos constituyen el $4 \%$ de la producción bruta industrial colombiana, en el año 2014 la producción bruta del sector se ubicó en 6.4 billones de pesos, es decir el 0,8 del PIB, siendo el subsector de fabricación de vehículos y motores el de mayor producción (67,3\%) seguido de autopartes (16,7\%) y carrocerías (16\%). Para este mismo año el sector aporto 18.3 mil empleos con un salario promedio mensual para los trabajadores del subsector de 2.4 millones de pesos [35]. Colombia es el cuarto productor de vehículos en Latinoamérica con 71.137 unidades en producción para el año 2014 y un mercado de 314.100 unidades vendidas en el mismo año [36].

Bogotá es la ciudad que más contribuye a la generación de empleo a nivel nacional. Durante el 2011, se presentó una tasa laboral de 2,5\% aumentando la tasa de ocupación con 1,4 puntos del 4,8\% nacional. Según [22] el sector ha sido el más importante motor de transferencia tecnológica para el desarrollo del país.

Los datos revelan la depresión que sufrió el sector desde 1998 hasta el 2003 [37], año en el que las ventas iniciaron un ascenso debido a las bajas tasas de interés del momento, a un repunte general de la economía y al fortalecimiento del peso, con excepción del año 2009, en donde las ventas disminuyeron, desde 2015 se ha presentado una baja en las ventas debido a los efectos de la devaluación, lo que ha impactado más el nicho de los importados [38].

En 2012, los países de destino de las exportaciones, en su orden son, México 43,17\%, Ecuador 28,80\%, Perú 9,96\%, Argentina 8,78\%, Chile $4,36 \%$, Venezuela 2,13\%, Guatemala 1,20\%, Pa- namá 1,20\% y los demás 1\%. Cabe mencionar que el $51 \%$ de las exportaciones colombianas se hacen a países que tienen unas industrias automotrices competitivas y fortalecidas, como México y Argentina. Los artículos del sector que más se exportan son vehículos de pasajeros, piezas de repuestos, vehículos comerciales y motocicletas, clasificados como productos de manufactura de tecnología intermedia [39].

La colocación de vehículos importados ocasionó una baja significativa de la participación de la industria nacional en el consumo de vehículos. Los países desde donde provienen principalmente las importaciones son México (27\%), Corea de Sur (21\%), China (11\%), Japón (7\%), India y Estados Unidos con el (6\%), Argentina (5\%), Brasil (4\%), Alemania y Ecuador (3\%), Tailandia (2\%), Reino Unido, España y Canadá por pequeños porcentajes de participación en la importación [36].

El sector genera empleo indirecto asociado a empresas aseguradoras, combustibles y autopartes, venta de insumos, empresas de conducción y talleres de mantenimiento, entre otros. En el país se ensamblan principalmente vehículos ligeros, pero también buses, camiones, motocicletas y vehículos multipropósito como camiones para la basura y ambulancias. A continuación, se caracteriza cada paso de la CA del sector automotriz en Colombia:

\section{Paso 1.}

Identificar las etapas, escalones y enlaces de la CA

La industria automotriz en Colombia se concentra en tres grandes ejes: ensamble de vehículos, producción de autopartes y ensamble de motocicletas. Las ensambladoras más importantes que concentran la mayor producción son General Motors Colmotores, Sofasa, Mercedes e Hino Motors Manufacturing. Con relación a la demanda, el $60 \%$ de los vehículos que se venden en el país son importados y el $40 \%$ son ensamblados en Colombia. El valor de las importaciones de motores para automóviles fue de $\$ 1.627$ millones de dólares en 2008, $\$ 1.266$ millones en 2009 y tuvo su mayor volumen en 2010 con $\$ 2.023$ millones de dólares [22].

\section{A. Eslabón de Aprovisionamiento}

En Colombia hay 60 proveedores de fabricantes de autopartes según información dada por ACOLFA (Asociación colombiana de fabricantes de autopartes), y estos venden a las ensambladoras del país. Para el año 2014 la ensambladora de Mazda efectúa el cierre de la Compañía Colombiana Automotriz (CCA) debido a la situación de la industria, producto de dificultades comerciales con los países vecinos Venezuela y Ecuador, y productivas, al sólo estar utilizando el $30 \%$ de la capacidad instalada (asociada únicamente a 500 empleados cuando se llegó a tener 1600) [40]. En Colombia también exis- 
te un amplio espectro de empresas autopartistas, de carácter nacional, mixto y extranjero, entre ellas algunas japonesas, canadienses, americanas, francesas, mexicanas y venezolanas [41], abastecedoras de piezas e insumos a fabricantes, ensambladores y distribuidores [42]. Los proveedores directos o de primer nivel son los encargados de abastecer una parte o algunos de los sistemas principales de un automóvil. En este nivel se sitúan empresas como: Bosch, Dana, Delphi Automotive, Johnson Controls Inc. y Lear Corporation. A nivel Nacional e internacional (SIA) y navieras son las encargadas de importar autopartes e insumos: Csav, China Jheping, MSC, Maersk, Humburg Zud.

\section{B. Marcas (ensambladoras)}

Según datos de la Cámara de la Industria Automotriz de la Andi, en Colombia hay ocho plantas de ensamblaje de carros y vehículos comerciales: Colmotores (Isuzu, Volvo y Chevrolet), Sofasa (Renault), Hino Motors Manufacturing (Marca Hino, Grupo Toyota), Carrocerías Non Plus Ultra (con marca propia y los CKD Volkswagen), Compañía de Autoensamble Nissan (marca Nissan), Navistrans, Daimler y Foton. El resto del parque industrial lo completan las empresas de motos: Auteco (Kawasaki, Bajaj, Kymco y KTM), Fanalca (Honda), Incolmotos (Yamaha), Suzuki, AKT (AKT y TVS), UM, Ayco, Jialing y Jincheng [43].

Sofasa distribuye vehículos livianos Renault (ensamble e importación), y sus proveedores locales de segundo nivel son: Icolbesto: Pastillas y discos de frenos; Gabriel: amortiguadores; Dana: sistemas de suspensión; MAC: Baterias; Elf: aceites; UMO: sistemas de escape; Industrias Metálicas JB: suministros metálicos; Vitro: vidrios; e Inorca: fabricación de cojinería.

Chevrolet GM Colmotores (ensambla e importa marcas como Isuzu, Suzuki, Volvo y Chevrolet), sus proveedores locales de segundo orden son: Icolbesto: Pastillas y discos de frenos; Gabriel: amortiguadores; Dana: sistemas de suspensión; MAC: baterías; Mobil: aceites; y Trimco: expertos en asientos automotrices.

\section{Eslabón de Producción}

El sector automotriz se rige por la norma NTC-ISO/ TS16949 para asegurar la calidad de los productos ofrecidos por la industria. La norma fue ideada por la industria automotriz de la IATF (International Automotive Task Force) para motivar las mejoras en la CA y certificar los procesos del negocio a nivel mundial.

En Colombia las licencias de ensamblaje son un proceso costoso que implica la firma de autorizaciones para las marcas ya existentes en Colombia y renovar una licencia de ensamblaje es también costoso.
En Colombia se producen dos tipos de vehículos: Vehículos Completos Importados (CBU Completely Built Up) completamente armados, se trae el carro completo para venta directa en el país, de esta manera se puede traer un carro de alta gama para ensamblar, en pocas cantidades no es rentable, los carros completos pagan entre el $15 \%$ y el $35 \%$ de arancel dependiendo el tipo de vehículo. Y vehículos ensamblados, Completamente Armados en Colombia (CKD Completely Knock Down) son automóviles traidos por kids desarmados para ensamblarlos en Colombia, con algún nivel de integración nacional, de esta forma se puede repetir el ensamblaje del mismo modelo muchas veces de manera eficiente con un precio beneficioso para el consumidor final y competitivo en el mercado, los carros de ensamble nacional son los carros de gama media de alto volumen y solo pagan entre el 0 y $5 \%$ de arancel. En la tabla 6 , se presentan las 8 Ensambladoras en Colombia de acuerdo a tipo de vehículos.

El 7 de febrero de 2012, GM Colmotores marcó un hito en la historia del sector automotriz, cuando realizó un acto de colocación de la primera piedra de la Zona Franca Industrial Colmotores (ZOFICOL), con la cual se inicia la transformación de la compañía, con el fin de convertirse en el primer fabricante de vehículos en Colombia. Esta iniciativa se dio de la mano el gobierno y en el marco del Programa de Transformación Productiva del Ministerio de Comercio, Industria y Turismo, con el cual se busca convertir a Colombia en una plataforma fuerte en la exportación de autopartes y vehículos. Se pretende hacer una inversión de 380 mil millones de pesos que se utilizarán en la planta de la zona franca y en la introducción de nuevas tecnologías, que ayudarán al desarrollo de nuevos productos y al fortalecimiento del sistema global de manufactura de la compañía. El plan de Reconversión Industrial reanudará el desarrollo tecnológico y busca generar cerca de 150 empleos especializados directos y 750 indirectos además de la posibilidad de expansión a mercados internacionales, lo que permitiría producir 60.000 nuevos vehículos cada año [44].

Haciendo algo de historia, en 1969, la Sociedad de Fabricación de Automotores (SOFASA) se establece en Colombia como ensambladora de vehículos de la marca Renault de origen francés con el fin de estimular el desarrollo del sector automotriz en Colombia. Siguiendo con las ensambladoras se encuentra Nissan, que llega a Colombia en 1960 como distribuidor, comercializando las marcas Jhon Deere y Nissan Forklift, luego en 1984 inicia la creación de talleres autorizados, para en 1997 pasa a convertirse en ensambladora de su marca en el país.

Por otro lado, se encuentra la marca Hino que representa al grupo Toyota en el mercado de camiones pesados y buses. La marca tiene desde 1998 el $51,2 \%$ de la representación de Daihatsu y en el 2001 el 50,1\% de las acciones de Hino. En 2008 abre su 
primera instalación industrial en América Latina con el fin de ensamblar camiones escogiendo a Colombia.

Navitrans es otra compañía colombiana que nace en 1958 con la distribución de vehículos, tractores y motores de la marca International Harvester. En el año 1992 inicia la importación de vehículos de la marca International y en el 2003 inaugura la planta de ensamble en Bogotá de la marca AGRALE.

Otro proyecto importante de ensambladoras en Colombia incluye a FOTON, empresa China ensambladora de camiones ligeros, con el apoyo de su socio local CORBETA, mediante una inversión inicial de USD 4 millones, genera cerca de 400 empleos directos. Daimler Chrysler tiene un proyecto de ensamble de chasis para buses apoyado con el autopartista DANA, con planta en Colombia, lo que generaría USD 4 millones en compras de autopartes colombianas [5].

Marcopolo - Superpolo es una empresa fabricante de carrocerías para buses (multinacional Brasileña) con una buena cantidad de exportaciones a mercados de la región, como Ecuador, Perú y Costa Rica, aspecto que ha generado cerca de 800 nuevos empleos; no se tiene contemplada como ensambla-

Tabla 6. Ensambladoras en Colombia de acuerdo a tipo de vehículos

\begin{tabular}{|c|c|c|}
\hline $\begin{array}{l}\text { Tipo de } \\
\text { vehículos }\end{array}$ & Ensambladoras en Colombia & Red de distribución \\
\hline \multirow{3}{*}{$\begin{array}{l}\text { Vehículos } \\
\text { ligeros }\end{array}$} & $\begin{array}{l}\text { General Motors. Colmotores. } \\
\text { Ensambla marca Chevrolet } \\
\quad \text { (Estadounidense ) }\end{array}$ & Cuenta con más de 100 concesionarios autorizados en todo el país. \\
\hline & Renault. Sofasa (Francesa) & $\begin{array}{c}\text { Bogotá, Cartagena, Barranquilla, Santa Marta, Montería, Medellín, Cúcuta, } \\
\text { Bucaramanga, Sogamoso, Pereira, Armenia, Buenaventura, Manizales, } \\
\text { Ipiales, entre otros. }\end{array}$ \\
\hline & $\begin{array}{l}\text { Compañía de Autoensamble } \\
\text { Nissan (Japonesa) }\end{array}$ & $\begin{array}{c}\text { Concesionarios y servicio postventa: } \\
\text { Bogotá, Manizales, Barranquilla, Armenia, Medellín, Cali, Barrancabermeja, } \\
\text { Cartagena, Bucaramanga, Cúcuta, Duitama, Ibagué, Neiva, Pasto, Pereira, } \\
\text { Popayán, Santa Marta, Tunja, Valledupar, entre otras. }\end{array}$ \\
\hline \multirow{5}{*}{$\begin{array}{l}\text { Vehículos } \\
\text { comerciales } \\
\text { de carga }\end{array}$} & $\begin{array}{l}\text { General Motors. Colmotores } \\
\text { Ensambla marca Chevrolet } \\
\quad \text { (Estadounidense) })\end{array}$ & Cuenta con más de 100 concesionarios autorizados en todo el país. \\
\hline & $\begin{array}{l}\text { Hino Motors Manufacturing } \\
\text { (Japonesa) }\end{array}$ & $\begin{array}{l}\text { A través de PracoDidacol están en Bogotá, Barranquilla,Medellín, } \\
\text { Bucaramanga,Yopal, Pereira, Cali, Ibagué, Pasto, Cartagena, Villavicencio, y } \\
\text { otros concesionarios en Cúcuta, Tunja, Valledupar y Montería. }\end{array}$ \\
\hline & $\begin{array}{l}\text { Navistrans (Ensamblador } \\
\text { Colombiano desde 2003con la } \\
\text { marca AGRALE) }\end{array}$ & $\begin{array}{c}\text { Presencia en } 14 \text { ciudades entre ellas Bogotá, Cali, Medellín, Barranquilla, } \\
\text { Pereira, Bucaramanga, Pasto entre otras. }\end{array}$ \\
\hline & Fotón (Compañía China) & $\begin{array}{l}\text { Concesionarios en Bogotá, Barranquilla, Medellín, Bucaramanga, Neiva, } \\
\text { Pereira, Cali, Ibagué, Pasto, Cartagena, Villavicencio, y Cúcuta. }\end{array}$ \\
\hline & $\begin{array}{l}\text { Daimler Chrysler } \\
\text { Representantes de las marcas } \\
\text { Mercedes-Benz y Freightliner }\end{array}$ & $\begin{array}{l}\text { Concesionarios en Bogotá, Barranquilla, Manizales, Medellín, Bucaramanga, } \\
\text { Pereira, Cali, Ibagué, Cartagena, Villavicencio, y Yopal. }\end{array}$ \\
\hline \multirow{5}{*}{$\begin{array}{l}\text { Buses de } \\
\text { pasajeros }\end{array}$} & $\begin{array}{c}\text { Carrocerías Non Plus Ultra } \\
\text { (Ensamblador Colombiano desde } \\
\text { 1992) (Ensambla Volkswagen } \\
\text { desde 1999). }\end{array}$ & Bogotá, Santa Marta, Montería, Medellín y Cali. \\
\hline & General Motors. Colmotores & En todo el país con distribuidores autorizados. \\
\hline & $\begin{array}{l}\text { Navistrans (Ensamblador } \\
\text { Colombiano) }\end{array}$ & Varias ciudades del país. \\
\hline & Fotón (Compañía China) & Varias ciudades del país. \\
\hline & $\begin{array}{l}\text { Daimler Chrysler } \\
\text { Representantes de las marcas } \\
\text { Mercedes-Benz y Freightliner }\end{array}$ & Varias ciudades del país. \\
\hline
\end{tabular}

Fuente: Elaboración propia con datos [5][36]. 
dora en Colombia y su función principal es elaborar carrocerías, diseño, producción y comercialización de equipos para transporte terrestre y de carga. Se nombra en este estudio porque es un eslabón de la CA al ensamblar los chasis de buses y camiones.

También existen otras carroceras como AGA dedicada al ensamble de buses desde 1977, ABC carrocerías de Colombia que fabrica furgones y estacas, al igual que Carrocerías Súper Busscar de Colombia quienes son fabricantes de carrocerías para el sistema de transporte masivo y Carrocerías Panamericana S.A.S., entre otras.

\section{Eslabón de Distribución}

Las marcas importadas provienen de los siguientes países: Alemania: Audi, BMW, Mercedes Benz, Volkswagen y Porsche. Estados Unidos: Chrysler, Ford y Jeep. Francia: Citroën y Peugeot. Japón: Dahiatsu, Honda, Mitsubishi, Nissan, Subaru y Toyota. Italia: Fiat, Ferrari y Maseratti. Corea: Hyundai, Kia y Ssangyong. Inglaterra: Jaguar y Land Rover. Suecia: Volvo. España: Seat [46]. En la tabla 7 se muestra los distribuidores autorizados [45].

Tabla 7. Distribuidores aUtorizados en Colombia POR MarCas

\begin{tabular}{|c|c|c|c|c|c|}
\hline Distribuidor & Marca & Distribuidor & Marca & Distribuidor & Marca \\
\hline Colitalia & $\begin{array}{l}\text { Alfa Romeo } \\
\text { Fiat }\end{array}$ & Audi Colombia & Audi & Sofasa & Renault \\
\hline Autogermana & $\begin{array}{l}\text { BMW } \\
\text { Mini }\end{array}$ & & Chevrolet & & $\begin{array}{c}\text { BYD } \\
\text { Daihatsu }\end{array}$ \\
\hline Autoelite & $\begin{array}{l}\text { Maserati y } \\
\text { Ferrari }\end{array}$ & Colmotores & $\begin{array}{l}\text { Isuzu } \\
\text { Suzuki } \\
\text { Volvo }\end{array}$ & Praco Didacol & $\begin{array}{l}\text { Hino } \\
\text { Mack } \\
\text { Peugeot } \\
\text { Subaru }\end{array}$ \\
\hline Parra Arango & Citroen & $\begin{array}{l}\text { Ford Motor de } \\
\text { Colombia }\end{array}$ & Ford & Fenalca & Honda \\
\hline $\begin{array}{l}\text { Hyundai } \\
\text { Colombia } \\
\text { Automotriz }\end{array}$ & Hyundai & Metrokia & Kia & Mazda de Colombia & Mazda \\
\hline $\begin{array}{l}\text { Distoyota } \\
\text { Toyota de } \\
\text { Colombia }\end{array}$ & $\begin{array}{c}\text { Toyota Importado } \\
\text { Toyota }\end{array}$ & Com. Automotriz & Changan & SK Bergé Colombia & $\begin{array}{c}\text { Chrysler } \\
\text { Jeep } \\
\text { Dodge } \\
\text { Ram }\end{array}$ \\
\hline Foton & Foton & Daimler Colombia & $\begin{array}{c}\text { Freightliner } \\
\text { Mercedes Benz }\end{array}$ & China Automotriz & Hafei - Yuejin \\
\hline Navitrans & International & Eurotrans & Iveco & $\begin{array}{c}\text { Grupo Premier } \\
\text { Motores Británicos }\end{array}$ & $\begin{array}{c}\text { Jaguar } \\
\text { Land Rover }\end{array}$ \\
\hline $\begin{array}{c}\text { Distribuidora } \\
\text { Changhe de } \\
\text { Colombia }\end{array}$ & JMC - Change & Industrias Ivor & Kenworth & $\begin{array}{l}\text { Distribuidora los } \\
\text { autos de Colombia }\end{array}$ & MG \\
\hline $\begin{array}{l}\text { Motores y } \\
\text { máquinas }\end{array}$ & Mitsubishi & $\begin{array}{l}\text { Distribuidora } \\
\text { Nissan }\end{array}$ & Nissan & Autoelite Ltda. & Porsche \\
\hline Vas Colombia & $\begin{array}{c}\text { Seat } \\
\text { Skoda }\end{array}$ & $\begin{array}{c}\text { SsangYong Motor } \\
\text { Colombia }\end{array}$ & SsangYong & Derco Colombia & Suzuki \\
\hline $\begin{array}{l}\text { Porsche de } \\
\text { Colombia }\end{array}$ & Volkswagen & $\begin{array}{l}\text { Automotriz } \\
\text { Escandinava }\end{array}$ & Volvo & Cinascar & $\begin{array}{l}\text { Zx Auto } \\
\text { Zotye } \\
\text { Chery }\end{array}$ \\
\hline
\end{tabular}




\section{E. Operadores logísticos}

Transporte y Logística HH, Uship, ABECE, ILT, Capital y Transportar son algunas de las empresas de transporte encargadas de llevar las autopartes desde los puertos hasta los concesionarios o distribuidores autorizados en cada ciudad del territorio colombiano.

Dentro de la CA también se encuentran operadores logísticos que suministran repuestos originales para algunas marcas, entre ellos están Andinas S.A., quienes suministran repuestos originales a nivel nacional para Mercedes Benz, Freightliner y Qingqi; Sibecol S.A.S., los cuales son representantes exclusivos para Colombia de las marcas Alemanas Norma, Stimberg, Beneri, Mekafix y W\&K, con sede en Bogotá y Barranquilla; Multikia Ltda., le distribuyen repuestos originales a Kia y Hyundai; y Autogrande que comercializa repuestos originales de todos los vehículos de Chevrolet, y cuenta con talleres de servicio autorizados [47].

Algunos de los operadores internacionales que apoyan el transporte de los automóviles hacia Colombia son: Csav, empresa dedicada a prestar servicios de transporte marítimo bimodal y servicios de enlace entre los diversos actores del comercio exterior; y China Jheping, que presta servicios de líneas regulares de contenedores y llegan normalmente al puerto de Buenaventura, entre otros importantes para la entrada de los automóviles a Colombia.

Luego de llegar a puerto, los automóviles son llevados al centro del país o a las principales ciudades a través de otros operadores logísticos como Logística $\mathrm{HH}$, quienes se dedican a prestar soluciones de transporte de vehículos y carga en general para fabricantes e importadores de vehículos y Transportar Cargo Logistics S.A.S., que es un agente de carga internacional.
Una representación de la operación de la CA de Automóviles para importación de vehículos terminados (ensamble fuera de Colombia) se presenta en la Fig. 1.

Paso 2. Describir el valor agregado de los agentes y componentes de la $C A$

\section{A. Eslabón de Aprovisionamiento}

Los proveedores indirectos o sub-proveedores se caracterizan por surtir componentes especializados. La mayoría de proveedores de segundo nivel también hacen parte del primer nivel. Estas empresas diseñan y producen para sus clientes ensambladores y requieren desarrollar e innovar permanentemente tanto en procesos como en productos. Se estima que los proveedores europeos de primer nivel tratan en promedio con 100 sub-proveedores [21].

\section{B. Eslabón de Producción}

Son los productores del bien final, asociados centralmente en Colombia con las ensambladoras. El proceso inicia en la gestión de mercadeo, donde se investigan las necesidades del mercado y se determinan los volúmenes y referencias a vender. La mayoría de piezas de ensamblaje llegan desde proveedores del exterior y algunas piezas del mercado local. Luego pasan a las áreas de soldadura, pintura y ensamble en donde se culmina el proceso de producción del vehículo bajo rigurosos estándares de calidad de acuerdo al modelo [48]. En términos de empleo, este es el eslabón más importante para el país, por soportar en buena parte el empleo indirecto, además del empleo directo, con buena calidad y estándares industriales. Adicionalmente, genera desarrollo industrial al suministrar una plataforma de desarrollo a los proveedores, algunos de ellos nacionales.

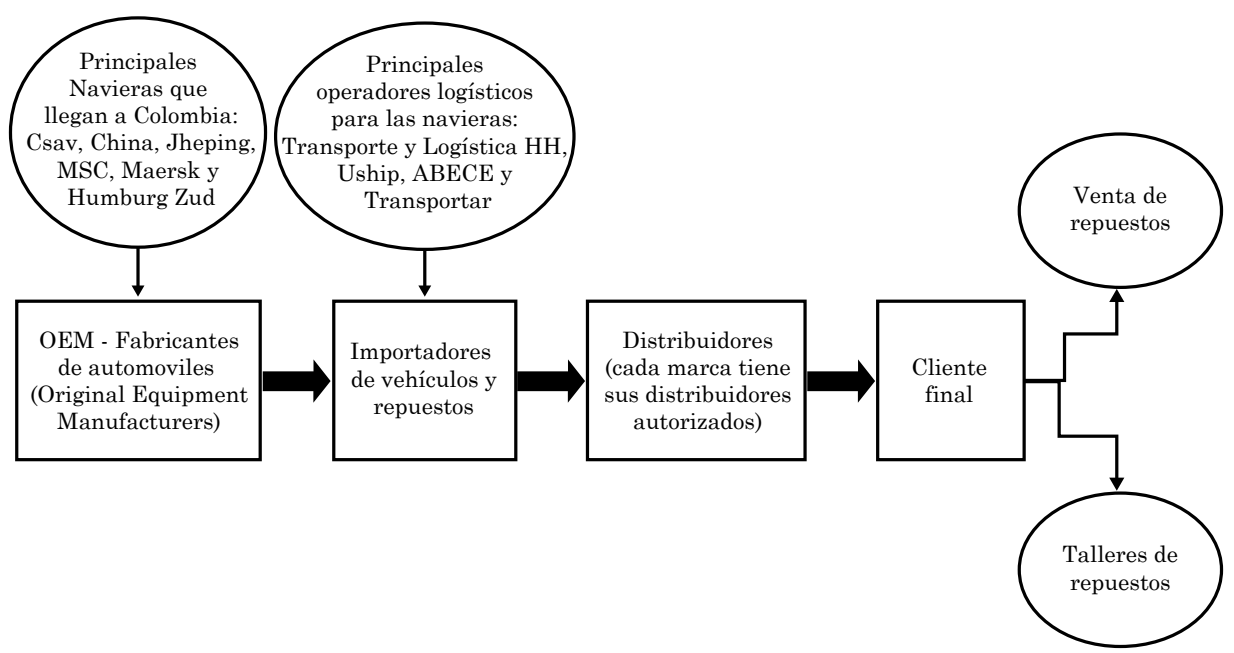

Fig. 1. Cadena de suministro para los automóviles Completely Built Up (CBU). Fuente: Elaboración propia. 


\section{Eslabón de Distribución}

$\mathrm{Su}$ valor agregado se centra en entregar en perfectas condiciones los vehículos $0 \mathrm{Km}$. La etapa se concreta en el trasporte de los vehículos desde la planta de ensamblaje hasta las empresas concesionarias; existen muchas variaciones en el modelo de distribución dentro de la industria automotriz, incluso los fabricantes más reconocidos usan modelos de distribución diferentes dependiendo de la región.

Colombia se ha convertido en una excelente plataforma exportadora para compañías extranjeras como Yasaki, Michelin, Saint Gobain, Goodyear, Dupont, Dana Corporation y Vitro, entre otras. La ubicación geográfica del país lo hace en un entorno logístico competitivo, por ser un paso obligado para las navieras y tener cercanía con el canal de Panamá, facilitando las interconexiones este-oeste, por poseer acceso a los dos océanos y tener un aeropuerto como el de Bogotá, considerado el primero en movimiento de carga a nivel latinoamericano.

La relación entre los fabricantes de autos y los distribuidores se da a través de concesiones o franquicias con relaciones empresariales a largo plazo, lo que supone una organización de sus funciones y tareas, con el fin de lograr ganancias conjuntas. En este sentido, existe una relación de dependencia entre las partes y fuentes de poder, al ser normalmente el fabricante quien tiene dominancia sobre los distribuidores y en esa medida los intermediarios dependen en gran parte del fabricante. Esta relación también se caracteriza por la cooperación y el conflicto, en donde se deben coordinar actividades para que las dos partes puedan lograr sus metas. La cooperación es necesaria entre los canales de distribución y el fabricante, y normalmente el conflicto surge por la dualidad entre la necesidad de los franquiciadores de controlar a sus franquiciados y la independencia de éstos como empresas jurídicamente diferentes [49].

\section{Cadena de abastecimiento de vehículos en Colombia}

En la Fig. 2 se presenta un esquema que muestra la relación entre las partes conformantes de la CA del sector automotriz en Colombia para automotores ensamblados dentro del país.

A manera de ejemplo, para el caso de los automóviles ensamblados en Colombia, la CA inicia con los OEM (Original Equipment Manufacturers), empresas fabricantes de automóviles y autopartes originales, quienes surten a los proveedores nacionales y extranjeros de primer y segundo nivel de acuerdo al país de origen. Un eslabón importante de la CA son las navieras al traer a los puertos las autopartes e insumos que llegan al país y son recibidos por las ensambladoras propias de las OEM o por las importadoras y distribuidoras. En puerto, las autopartes e insumos son llevadas a los concesionarios y talleres a través de operadores logísticos como Transporte y Logística HH, Uship, ABECE y Transportar, entre otros.

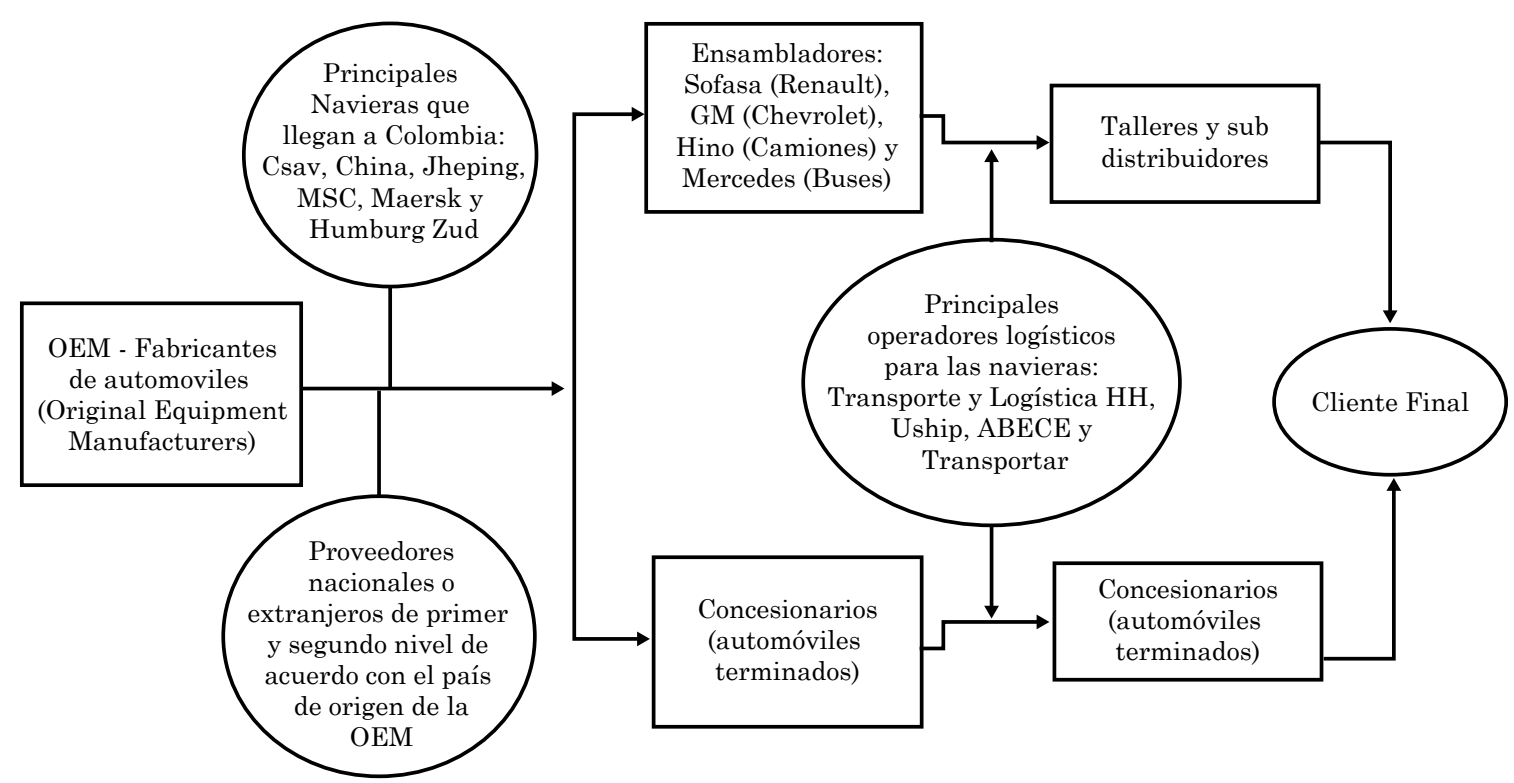

Fig 2. Cadena de abastecimiento del sector automotriz en Colombia para automotores CKD. Fuente: Elaboración propia. 
Paso 3. Describir las especificidades del desempeño de la $C A$

La venta de autos nuevos en Colombia tuvo su pico más alto en diciembre de 2014 con un aumento del $25 \%$ comparado con el mismo mes en el año 2013. En la feria Internacional del automóvil, llevada a cabo en noviembre de 2014, el total de ventas de autos nuevos supero en un $12 \%$ al año previo. Lo que muestra un aumento en la comercialización de vehículos, aspecto motivante para el sector industrial y de autopartes. El $75 \%$ del parque automotor le pertenece a losestratos 2,3 y 4 , este proceso de masificación ha hecho que la producción de carros haya ido en aumento, en un $6,1 \%$ con relación al año 2013 [50].

Bogotá es la ciudad con mayor venta de carros nuevos, seguida de Cali, Envigado y Medellín. Dentro de las estadísticas ofrecidas por Asopartes los vehículos importados más vendidos son automóviles, seguidos de los carros utilitarios, los pick ups y los taxis, y en el mismo orden pero con un porcentaje de venta más bajo están los carros ensamblados en Colombia [50].

El empleo en el sector automotor y de autopartes es 3.5 veces mejor pagado que el promedio del pago de un trabajador de otra industria. Cabe destacar también el esfuerzo de las compañías de automóviles por capacitar a su personal. Los empleos más solicitados por el sector son: supervisores mecánicos, soldadores, mecánicos industriales, mecánicos de equipo pesado, electricistas de vehículos, latoneros, técnicos en pintura, coloristas y vendedores, entre otros [50]. Una de las grandes ventajas de Colombia es tener "hubs" o centros de distribución para cubrir los mercados de Latinoamérica y el Caribe; adicional a esto, existe presencia de 4 megacarrier (navieras más grandes del mundo), el $75 \%$ del total de las navieras en el mundo arriban en servicios de importación y exportación, tienen más de 3.700 frecuencias marítimas, bajos costos en fletes marítimos para el transporte de carga hacia Norteamérica, Suramérica y Centroamérica y 18 aerolíneas de carga y 13 de pasajeros que prestan sus servicios en el país [5].

\section{Paso 4. Diagnóstico y conclusiones sobre la condición de la $C A$}

En Colombia se ha venido experimentando un proceso de deslocalización. Pese al crecimiento de Colmotores y la buena posición de Renault, que aumentaron la producción, han venido perdiendo participación del mercado. El mercado nacional es relativamente pequeño y no se ha logrado una relación comercial más amplia a nivel región debido a dificultades políticas, esto ha generado el proceso de deslocalización que llevo al cierre de la CCA.
En esta CA, el exportador es en muchas ocasiones el mismo fabricante, quien produce en otro país, e ingresa los productos a través de un importador, quien los distribuye a mayoristas o ensambladoras en Colombia y a su vez distribuyen el producto a los minoristas.

Los mayores proveedores de autopartes en Colombia se discriminan por marcas. La concentración de proveedores de autopartes está más en el segundo nivel, que son los importadores de autopartes, con un $75 \%$ de participación en el mercado y los distribuidores de vehículos con un $25 \%$ de participación con el manejo de repuestos.

Canadá y Estados Unidos son vistos como demandas de oportunidad, para exportar materiales de construcción, autopartes y plásticos, facilitado por el TLC entre Colombia y Canadá, el acuerdo comercial ATPDEA con Estados Unidos y los estímulos del gobierno que estimulan el sector buscando una mayor dinamización de estos sectores [51].

De acuerdo con los errores o fallos más comunes encontrados en las CAs [52] a causa de razones regulatorias y errores en infraestructura, es importante afirmar que dichos errores dentro de la CA son vistos como la variación en la distribución de los resultados de la CA y como eventos no planificados e imprevistos que interrumpen el flujo normal de los bienes y materiales, teniendo en cuenta lo anterior, los principales problemas de la CA en Colombia están relacionados con el cubrimiento a nivel nacional y el servicio postventa [53].

La CA verde se ha convertido en una parte importante en las empresas teniendo en cuenta la necesidad de cuidar el planeta y manejar adecuadamente los recursos, aspecto que generan diferencias competitivas en la CA. Según [54] el estudio de las estrategias ambientales orientadas a la sostenibilidad del medio ambiente aplicadas a la CA, significa un reto para Colombia en la medida en que debe utilizar el conocimiento y la información generadas a partir de las evaluaciones ambientales para promover la aplicación de las CAs verdes en el país, incluida la de automotores que es reconocida como una de las que mayor impacto ambiental genera. En este sentido, aunque ha habido avances en términos de la regulación sobre mínimos de emisiones de $\mathrm{CO}_{2}$ hay mucho trabajo aún por hacer para cerrar la brecha respecto a los países del primer mundo. Haciendo referencia a estos esfuerzos, el Decreto 948 de 1994 prohíbe el uso de combustible contaminante, particularmente de aceites lubricantes gastados y otros residuos considerados peligrosos, establece la necesidad de que las empresas cuenten con un plan de contingencia cuando tienen emisiones riesgosas para la salud, establece la obligación de obtener permiso de emisión de fuentes fijas, define la obligación de cumplir normas de emisiones (D. 02/82) y obliga a presentar informes de estado de emisiones cuando la reglamente el Ministerio 
del Medio ambiente. Así mismo, la Ley 430 de 1998 regula todo lo relacionado con la prohibición de introducir desechos peligrosos al territorio nacional, en cualquier modalidad, y con la responsabilidad por el manejo integral de los generados en el país y en el proceso de producción, gestión y manejo de los mismos, así mismo regula la infraestructura de la que deben ser dotadas las autoridades aduaneras y zonas francas y portuarias, con el fin de detectar de manera técnica y científica la introducción de estos residuos, regula las sanciones en la Ley 99 de 1993 para quien viole el contenido de esta Ley y se permite la utilización de los aceites lubricantes de desechos, con el fin de producir energía eléctrica. Así, el productor de residuos peligrosos y la entidad que contrate para la prestación del servicio son responsables por los efectos ambientales y a la salud pública generada por la producción, recolección, manejo y disposición final.

Con relación a las estrategias ambientales orientadas a la sostenibilidad del medio ambiente aplicadas a la CA [54], en Colombia las importadoras de vehículos completos pueden importar desde carros eléctricos hasta carros híbridos, con un número limitado de carros importados al año, los cuales gozan de $100 \%$ de exenciones arancelarias. Esas son iniciativas del gobierno con el tema ambiental. En autopartes, el tema de baterías y lubricantes tiene control para su disposición final; y con relación a las leyes ambientales, como la política para el Manejo Integral de los Residuos de 1997 del Ministerio del Medio Ambiente, la cual busca impedir o minimizar los riesgos para los seres humanos y el medio ambiente, ocasionados por los residuos sólidos y peligrosos, y minimizar la peligrosidad en la disposición final.

\section{Discusión}

La industria colombiana requiere transformaciones de fondo para poder enfrentar cambios tecnológicos y la fuerza de la competencia mundial [55]. De acuerdo con estos planteamientos [56] existen problemas en la optimización de las CAs de suministro orientados hacia dos niveles: las decisiones estratégicas relacionadas con la localización, el número y la capacidad de las instalaciones, y las decisiones tácticas y operativas relacionadas con la política de ensamblaje, los niveles de inventario y la programación. En particular, el modelo propuesto por los autores hace hincapié en la importancia de la flexibilidad táctica a la hora de tomar decisiones estratégicas. Con relación a lo anterior, la CA en Colombia presenta problemas de optimización en las decisiones estratégicas, debido a la falta de atención y repuestos en la red, fallas en el cubrimiento a nivel nacional y el servicio postventa. Esta barrera de entrada se debe principalmente al nivel económico de las empresas, debido a que la instalación de una red postventa requiere inversiones muy altas de dinero, tiempo y experiencia en el mercado [53], lo que plantea retos organizacionales y gubernamentales para el fomento a la industria.

A partir de [57] se busca medir la flexibilidad de los eslabones de la CA respecto a cambios de la demanda del mercado. En Colombia, el incentivo que propicia el funcionamiento de la CA se sustenta en el potencial de rentabilidad para los importadores de repuestos, el cual es alto en términos de márgenes brutos y utilidades, aunque requiere inversiones importantes donde la incidencia del cambio del dólar es baja porque la demanda es inelástica; las personas pagan el precio por tener la autoparte que requieren para su vehículo. En los otros eslabones de la CA quedan ganancias menos conocidas de acuerdo a las negociaciones y cubrimiento contractual con los proveedores [53].

\section{Conclusiones}

Los volúmenes de venta de carros en Colombia nunca han llegado a un volumen suficiente como para que haya más ensambladoras, en esa medida resulta más económico comprar un carro ensamblado en su lugar de origen, en donde tienen una economía a escala y el carro a pesar de pasar por tantos aranceles es más económico que traerlo y ensamblarlo en Colombia. Lo que determina traer el vehículo completo o ensamblarlo al país es el volumen de ventas del modelo que se va a traer, y la economía del arancel que sopesa con el costo de ensamblarlo localmente.

Las empresas ensambladoras en el país a la hora de escoger sus proveedores se decantan por criterios productivos. No obstante, la competencia entre los proveedores se fundamenta en una mayor cantidad de aspectos incluyendo: calidad del producto, plazo y puntualidad de la entrega, mejor precio, mayor facilidad para adaptarse a la empresa ensambladora y mayores niveles de integración tecnológica.

En el nivel secundario, la parte informal es muy grande y proveen muchas autopartes, en repuestos el control de la franquicia es muy difícil de tener; es común que las fábricas tengan varias franquicias debido a la dificultad de mantener cobertura a nivel nacional.

El principal reto de la distribución de las marcas de importados en Colombia es el soporte de producto, servicio postventa y repuestos en toda la geografía nacional. Hay marcas que fallan en el cubrimiento a nivel nacional, venden muy bien en ciudades principales del país mientras en ciudades intermedias o pequeñas no hay cubrimiento. Al respecto, el problema no solo se centra en las dificultades de transporte del eslabón logístico, también es afectado por el desarrollo del soporte de talleres especializados, personal capacitado e inventario suficiente [53]. 


\section{AgRadecimientos}

Los autores agradecen a la Universitaria Agustiniana, la Universidad Pedagógica y Tecnológica de Colombia y a la Corporación Minuto de Dios, por el soporte para el desarrollo del trabajo.

\section{REFERENCIAS}

[1] R. G. García-Cáceres, A. Perdomo, O. Ortiz, P. Beltrán and K. López, "Characterization of the supply and value chains of Colombian cocoa", Dyna, vol. 81, no. 187, pp. 30-40, Oct. 2014. https://doi.org/10.15446/dyna. v81n187.39555

[2] S. Chopra and P. Meindl, Supply Chain Management. New Jersey, USA: Prentice Hall, 2007.

[3] M. Ortega, "Cadena de suministro, reto automotriz", in Expansión, Jul. 2014. [Online]. Recuperado de https:// expansion.mx/opinion/2014/07/16/automotrices-consumen-menos-suministros

[4] A. Prakash, A. Agarwal and A. Kumar, "Risk Assessment in Automobile Supply Chain”, Mater. Today Proc., vol. 5, no. 2, pp. 3571-3580, Jan. 2018. http://dx.doi. org/10.1016/j.matpr.2017.11.606

[5] Incentivos del sector automotriz. Colombia 2016, ProColombia, Bogotá, D.C., 2016.

[6] R. G. García-Cáceres and É. S. Olaya, "Caracterización de las cadenas de valor y abastecimiento del sector agroindustrial del café", Cuad. Adm., vol. 19, no. 31, pp. 197-217, Jun. 2006.

[7] R. García-Cáceres, A. Núñez-Moreno, T. Ramírez-Ortiz and S. Jaimes-Suárez, "Caracterización de la fase Upstream de las Cadenas de Valor y de Abastecimiento de Palma de Aceite en Colombia," Dyna, vol. 179, pp. 7989, Jun. 2013

[8] R. B. Stone and K. L. Wood, "Development of Functional," vol. 122 , no. 4, pp. 360-370, Dec. 2000. http://dx.doi. org/10.1115/1.1289637

[9] J. Hirtz, R. B. Stone, D. A. McAdams, S. Szykman and K. L. Wood, "A functional basis for engineering design: Reconciling and evolving previous efforts", Res. Eng. Des., vol. 13, no. 2, pp. 65-82, Feb. 2002.

[10] R. G. García-Cáceres and J. W. Escobar, "Caracterización de las problemáticas de la cadena de abastecimiento," Dyna, vol. 83, no. 198, p. 68-78, Jun. 2016. https://doi.org/10.15446/dyna.v83n198.44532

[11] N. Yepes, E. López, C. Quintero and J. J. González, "Factores que impactan en la selección e implantación del sistema de gestión ISO/TS 16949: caso del sector industrial de autopartes de la ciudad de Bogotá", Cienc. e Ing. Neogranadina, vol. 24, no. 1, pp. 143-162, Jun. 2014. https://doi.org/10.18359/rcin.12

[12] P. Aguilar-Pérez and L. P. Cruz-Covarrubias, "Esquema de condicionantes en la relación proveedor-cliente en la industria automotriz . Caso sector autopartes en la Zona del Bajío", Rev. Ing. y Organ., no. 56, pp. 57-67, 2015.

[13] T. Kito and K. Ueda, "The implications of automobile parts supply network structures: A complex network approach”, CIRP Ann. - Manuf. Technol., vol. 63, no. 1, pp. 393-396, Dec. 2014. https://doi.org/10.1016/j. cirp.2014.03.119

[14] S. Luthra and S. Kumar, "When strategies matter: Adoption of sustainable supply chain management practices in an emerging economy's context", Resour. Conserv. Recycl., vol. 138, no. 1, pp. 194-206, Nov. 2018. http://dx.doi.org/10.1016/j.resconrec.2018.07.005

[15] T. E. González and M. A. Martin, "La innovación en entornos económicos poco favorables: el sector auto partes mexicano," Estud. Gerenciales, vol. 29, no. 127, pp. 167-176, Jun. 2013. https://doi.org/10.1016/j.estger.2013.05.005
[16] Y. Carbajal, B. Carrillo and L. De Jesus, "Dinámica productiva del sector automotriz y la manufactura en la frontera norte de México : Un análisis con datos de panel, 1980-2014", Front. Norte, vol. 30, no. 59, pp. 2956, Jun. 2017. http://dx.doi.org/10.17428/rfn.v30i59.511

[17] L. F. Scavarda, P. S. Ceryno, S. Pires and K. Klingebiel, "Supply Chain Resilience Analysis: a Brazilian Automotive Case," Rev. Adm. Empres., vol. 55, no. 3, pp. 304-313, Jun. 2015. http://dx.doi.org/10.1590/ S0034-759020150306

[18] M. Cedillo-Campos and C. Sánchez-Ramírez, "Dynamic Self-Assessment of Supply Chains Performance: an Emerging Market Approach," J. Appl. Res. Technol., vol. 11, no. 3, pp. 338-347, Jun. 2013.

[19] P. E. O. de Castillo, M. Vivaldi, S. R. I. Pires, A. R. T. Terra Argoud and J. B. de Camargo Junior, "La Gestión de Proveedores de Segundo Nivel en la Cadena de Suministros. Un Estudio en la Industria Automotriz en Brasil," Invenio, vol. 18, no. 34, pp. 109-118, Jun. 2015 .

[20] "World Motor Vehicle Production", OICA (Organisation Internationale des Constructeurs d'Automobiles). [Online]. Available: http://www.oica.net/category/production-statistics/2017-statistics/

[21] J. Jiménez, "Un análisis del sector automotriz y su modelo de gestión en el suministro de las autopartes", IMT, San Fandila, Publicación técnica No. 288, 2006.

[22] PNUD, "El sector automotor: Oportunidades de inclusión productiva para poblaciones en pobreza en Bogotá”, 2013. [Online]. Disponible en https://info.undp. org/docs/pdc/Documents/COL/00058568_Estudio\%20 de\%20Perfiles\%20Sector\%20Automotor_Baja.pdf

[23] R. Basurto, "Estructura y recomposición de la industria automotriz mundial, oportunidades y perspectivas para México", Rev. Econ. UNAM, vol. 10, no. 30, pp. 75-92, Dec. 2014. https://doi.org/10.1016/S1665952X(13)72204-7

[24] S. Medina, "¿Resurge la industria automotriz de Estados Unidos?”, Comer. Exter., vol. 62, no. 2, pp. 18-21, Mar. 2012.

[25] Associated Press, "Gas prices put Detroit Big Three in crisis mode", in NBC NEWS, Jan. 2008. [Online]. Available: http://www.nbcnews.com/id/24896359/ns/ business-autos/t/gas-prices-put-detroit-big-three-crisis-mode/

[26] J. E. Mendoza, "El impacto de la crisis automotriz," Nueva época, vol. 20, no. 2, p. 281-309, Jan. 2011.

[27] Revista Motor, "El 'chispazo' de Tesla lo convirtió en símbolo mundial de la movilidad eléctrica", in El Tiempo, May. 2015. [Online]. Disponible en https://www.eltiempo.com/archivo/documento/CMS-12829545

[28] D. Salazar-Carreño, R. G. García-Cáceres and O. O. Ortiz-Rodríguez, "Laboratory processing of Colombian rice husk for obtaining amorphous silica as concrete supplementary cementing material", Constr. Build. Mater., vol. 96, pp. 65-75, Oct. 2015.

[29] M. Dijk and M. Yarime, "The emergence of hybrid-electric cars: Innovation path creation through co-evolution of supply and demand", Technol. Forecast. Soc. Change, vol. 77, no. 8, pp. 1371-1390, Oct. 2010. https://doi. org/10.1016/j.techfore.2010.05.001

[30] Revista Motor, "Top 10 de las marcas que más venden en el mundo", El Tiempo, Apr. 2017. [Online]. Diponible en https:/www.motor.com.co/actualidad/industria/top10-marcas-venden-mundo/29031

[31] C. Coba, "Ranking de los 10 países con más autos por habitante", Seguros 123, Feb. 2015. [Online]. Disponible en https://ecuador.seguros123.com/ranking-delos-10-paises-con-mas-coches-por-habitante/

[32] Revista Latin America Hoy, "El sector automotriz en América Latina”, Latín América Hoy, Apr. 2012. Recuperado de https://latinamericahoy.es/2012/04/17/elsector-automotriz-en-america-latina/ 
[33] N. Meza, "Los 10 países con mayor producción de autos en el mundo", Forbes, May. 2015. [Online]. Disponible en http://www.forbes.com.mx/los-10-paises-con-mayorproduccion-de-autos-en-el-mundo/

[34] K. Hayashi and T. Nemoto, "Procurement Logistics of Japanese Auto Manufacturers in Inland China", Asian J. off Shipp. Logist., vol. 26, no. 1, pp. 119-138, Jun. 2010. https://doi.org/10.1016/S2092-5212(10)80014-8

[35] BBVA, "Situación automotriz Unidad de Colombia," Research, 2016. [Online]. Disponible en https://www.bbvaresearch.com/wp-content/uploads/2016/04/2.-SectorAutomotor-y-Motos.pdf

[36] ANDI, "Informe del Sector Automotor a septiembre de 2014". [Online]. Disponible en https://es.slideshare.net/ HansLamprea/informe-del-sector-automotor-a-septiembre-2014-andi-fenalco

[37] Asopartes, "Informe ventas 2013". [Online]. Recuperado de https://asopartes.com/es/mision-vision-politicasdecalidad/estadisticas-del-sector/category/8-ano-2013

[38] J. A. Rivera-Godoy y A. M. Padilla-Ospina, "Sector autopartes en Colombia: comportamiento financiero durante el período 2008-2014", Entramado, vol. 23, no. 1, pp. 12-29, Jun. 2016. http://dx.doi.org/10.18041/ entramado.2016v12n1.23113

[39] Mincit, "Informes de exportación", Oficina de Estudios Económicos, 2014. [Online]. Disponible en http://www. mincit.gov.co/estudios-economicos/estadisticas-e-informes/informes-de-exportacion

[40] H. Rodríguez, "El fin de la ensambladora Mazda", in Dinero, Oct. 2014. [Online]. Disponible en https://www. dinero.com/empresas/articulo/mazda-no-ensamblaracolombia/191801

[41] C. Bonilla, "Autopartistas colombianos: en la lucha", Internacional Metalmecánica, Aug. 2012. [Online]. Disponible en http://www.metalmecanica.com/temas/Autopartistas-colombianos,-en-la-lucha+7088946

[42] J. A. Zapata, A. F. Álvarez and S. Ruiz, "Caracterización del sector autopartes- automotor en Colombia”, Rev. Escenarios Empres. y Territ., no. 2, pp. 335-354, Dec. 2013.

[43] V. Pérez, "Por cierre de la CCA, autos que se vendan de Mazda serán solo importados", La República, May. 2014. [Online]. Disponible en https://www.larepublica. co/empresas/por-cierre-de-la-cca-autos-que-se-vendande-mazda-seran-solo-importados-2116806

[44] G. Bonilla, "GM Colmotores se convierte en el primer fabricante de vehículos en Colombia", F1, Feb. 2012. [Online]. Disponible en http://www.f1latam.com/autos/ noticias.php?idn $=479$

[45] Revista Motor, "Distribuidores". El Tiempo, Jun. 2015. [Online]. Disponible en http://app.motor.com.co/revistamotor/uploads/files/2015/06/04/nuevos_final\%20-2-.pdf

[46] G. Avendaño, "Descubra el origen de los vehículos que se comercializan en Colombia", El Tiempo, Aug. 2008. Disponible en https://www.motor.com.co/actualidad/ lanzamientos/descubra-origen-vehiculos-comercializancolombia/2079

[47] Directorio empresarial colombiano, "Operadores logísticos” Apr. 2015. [Online]. Disponible en https://directorioempresarialcolombiano.com/es/operadores-logisticos. html

[48] Renault, "Renault Servicios", Renault.com, 2015. [Online]. Disponible en https://www.renault.com.co/servicios/renault-servicios.html

[49] J-P. Mangin, V. Apaolaza y P. Hartmann, "Determinantes del éxito de las relaciones fabricante-distribuidor: el caso de los concesionarios de automóviles estadounidenses en España," Cienc. Ergo Sum, vol. 14, no. 1, pp. $125-134$, Oct. 2007.

[50] Assopartes, "Informe ventas 2014". [Online]. Recuperado de https://asopartes.com/es/mision-vision-politicasdecalidad/estadisticas-del-sector/category/9-ano-2014

[51] L. G. Restrepo, R. Moseres y Stellabatti, Oportunidades en tiempos de diversificación e innovación, Bogotá, D.C.: Proexport, 2010.
[52] G. A. Zsidisin, B. N. Petkova and L. Dam, "Examining the influence of supply chain glitches on shareholder wealth: Does the reason matter?", Int. J. Prod. Res., vol. 54, no. 1, pp. 69-82, Feb. 2016. https://doi.org/10.1080/0 0207543.2015.1015751

[53] ComunicarSe, "Evalúan las materias primas y sus riegos en la industria automotriz", Cadena de valor. May. 2018. Disponible en https://www.comunicarseweb.com/ noticia/evaluan-las-materias-primas-y-sus-riegos-en-laindustria-automotriz

[54] A. Lake, A. Acquaye, A. Genovese, N. Kumar and S. C. L. Koh, "An application of hybrid life cycle assessment as a decision support framework for green supply chains", Int. J. Prod. Res., vol. 53, no. 21, pp. 6495-6521, Sep. 2015. https://doi.org/10.1080/00207543.2014.951092

[55] Revista Portafolio, "Industria automotriz nacional tiene futuro", Portafolio, Aug. 2010. Disponible en https:// www.portafolio.co/economia/finanzas/industria-automotriz-nacional-futuro-411858

[56] N. Hamta, M. A. Shirazi, S. M. T. F. Ghomi and S. Behdad, "Supply chain network optimization considering assembly line balancing and demand uncertainty", Int. J. Prod. Res., vol. 53, no. 10, pp. 2970-2994, Dec. 2014. https://doi.org/10.1080/00207543.2014.978030

[57] Y. Xiao, "Flexibility measure analysis of supply chain," Int. J. Prod. Res., vol. 53, no. 10, pp. 3161-3174, May. 2015. https://doi.org/10.1080/00207543.2014.975864

Gilma Rocio Peña Meneses es Psicóloga con Magister en Educación del Tecnológico de Monterrey (México) y Especialista en Psicología Educativa de la Universidad Católica (Colombia). Cuenta con 15 años de experiencia en educación superior y 5 años en investigación en la Universitaria Agustiniana (Colombia). https://orcid.org/0000-0002-0179-3319

Rafael Guillermo García Cáceres es Ingeniero Industrial de la Universidad Pedagógica y Tecnológica de Colombia-UPTC (Colombia), Doctor en Ingeniería de la Universidad de los Andes (Colombia) y Magister en Ingeniería Industrial de la Universidad de los Andes (Colombia), Profesor asociado en la UPTC (Colombia). Comisionado de la Sala de Ingeniería, Industria y Construcción del 2016 al 2019 y miembro de la Comisión Nacional de Aseguramiento de la calidad de la Educación Superior (CONACES). https://orcid.org/0000-0003-0902-1038 